\title{
DIFFERENTIABILITY PROPERTIES OF SOLUTIONS TO HIGHER ORDER DOUBLE INTEGRAL VARIATIONAL PROBLEMS $\left({ }^{1}\right)$
}

BY

JULIUS SMITH

Introduction. We confine our remarks to a summary of the contents of the paper. 'A brief history of the problem together with a bibilography may be found in [3].

In $\$ 0$ we collect notation, definitions and some theorems used in the remainder of the paper, including an existence theorem for the variational problem (Theorem 0.1).

In $\$ 1$ we show that if $\Delta^{m} u=0$ in $\overline{B_{r}}$, a closed disk of radius $r$

$$
\left(\Delta=\left(\partial / \partial x^{1}\right)^{2}+\left(\partial / \partial x^{2}\right)^{2}\right)
$$

then $u$ satisfies

$$
\int_{B_{r}}\left|\nabla^{m} u\right|^{2} d x \leqq l(m) r \int_{\partial B_{r}}\left|\nabla^{m} u\right|^{2} d s, \quad l(m)=2^{m}-1 .
$$

$\left(\nabla^{m} u=\left\{D_{1}^{\alpha_{1}} D_{2}^{\alpha} u\right\}, \alpha_{1}+\alpha_{2}=m,\left|\nabla^{m} u\right|^{2}=\right.$ weighted sum of squares of components of $\nabla^{m} u$ (see $\left.\$ 0\right) ; D_{j}=\left(\partial / \partial x^{j}\right)$.)

This estimate may be obtained easily for $m=1$, from a direct comparison of Fourier coefficients. Direct computation succeeds for $m=2$ but the complication increases with $m$ and this approach seems to fail for general $m$. If a function $f$ on $\bar{B}_{r}$ allows the expansion,

$$
f \sim a_{0}(r) / 2+\sum_{n=1}^{\infty} a_{n}(r) \cos n \theta+b_{n}(r) \sin n \theta,
$$

then in the usual way we define the conjugate function

$$
\bar{f} \sim a_{0}(r) / 2+\sum_{n=1}^{\infty} b_{n}(r) \cos n \theta-a_{n}(r) \sin n \theta .
$$

A more esthetically pleasing definition results if we consider only functions for which $a_{0}(r) \equiv 0$, but this is not necessary for our purposes. With the aid of Green's Theorem and some lemmas concerning the conjugate function which are of some interest in themselves, we prove (*) by in-

Received by the editors March 24, 1964.

( $\left.{ }^{1}\right)$ The research reported on in this paper was partially supported by contract NONR 222 (62) between the Office of Naval Research and the University of California at Berkeley. It forms a part of the author's doctoral dissertation written under the direction of Professor C. B. Morrey, Jr., to whom the author is indebted for many valuable suggestions. 
duction. The remainder of the paper offers a treatment parallel to that in $[3]$.

In §2 we introduce Morrey's lemma on Dirichlet growth which asserts that a family of functions whose Dirichlet integral $\left(\mathcal{\nabla}|\nabla u|^{2} d x\right)$ is at most $A r^{2 \lambda}(0<\lambda<1)$ over disks of radius $r$ and all sufficiently small $r$, satisfies a uniform Hölder condition on compact sets. (See Lemma 2.1 for a precise statement.) This is easily applied to prove a similar theorem if $\int\left|\nabla^{m} u\right|^{2} d x \leqq A r^{2 \lambda}$ (Lemma 2.2), and it is then possible to conclude that $\nabla^{m-1} u$ is uniformly Hölder continuous on compact sets.

With the aid of $(*)$ we extend to the case of arbitrary $m$ a condition sufficient to insure "Dirichlet growth" (Lemma 2.3; compare [1, Theorem 6.1]). This leads easily to our first regularity result concerning variational problems for double integrals whose integrands depend on derivatives of order $m$ (see (2.8) and Theorem 2.1). The remainder of $\$ 2$ deals with uniformly elliptic systems of equations in integrated form (see Definition 2.1 for conditions). Uniform growth properties are established in preparation for their application to the variational problem, hence no regularity is assumed for the coefficients since none can be expected for the coefficients arising from the variational problem.

Finally, in $\$ 3$ a difference quotient procedure is applied to the integrated form of the Euler-Lagrange equations. The difference quotients are shown to satisfy a system of elliptic equations with uniform bounds. Solutions to the variational problem are then shown to have Hölder continuous $m$ th derivatives on interior domains (Theorem 3.2). While this still is a step shy of the classical Euler-Lagrange equations, further results may be obtained by applying the continuity method to the integrated equations whose coefficients are now Hölder continuous (for $m=1$ see, e.g., [2]).

0 . Notation and background. $E_{\nu}$ is $\nu$-dimensional Euclidean space with points $x=\left(x^{1}, x^{2}, \ldots, x^{2}\right)$. In any theorem or lemma $\nu=2$ except if $\nu$ appears explicitly. $D$ or $G$ is a bounded domain in $E_{\nu}$ (open connected set) with boundary $\partial G$ and $\bar{G}=G \cup \partial G ; B(x, r)=$ open ball of radius $r$ and center $x$ in $E_{v}$ ( $B_{r}$ if no confusion arises); $u$ is generally a vector or tensor function $\left(u^{1}, \cdots, u^{N}\right) ; C^{m}(G)=$ functions $u$ continuous together with $m$ derivatives in $G(m=0, \ldots, \infty) ; C^{m}(\bar{G})=$ functions $u$ in $C^{m}(G)$ extendable to $C^{m}(D)$ for some $D \supset G ; \alpha=\left(\alpha_{1}, \alpha_{2}, \cdots, \alpha_{\nu}\right), \alpha_{i}$ a non-negative integer.

$$
D^{\alpha}=D_{1}^{\alpha_{1}} D_{2}^{\alpha_{2}} \ldots D_{\nu}^{\alpha_{\nu}}, \quad D_{j}=\frac{\partial}{\partial x^{j}}, \quad|\alpha|=\alpha_{1}+\alpha_{2}+\cdots+\alpha_{v} ;
$$

for $\nu=2$,

$$
D_{\rho \theta}^{\alpha}=D_{\rho}^{\alpha_{1}} D_{\theta}^{\alpha_{2}}, \quad D_{\rho}=\frac{\partial}{\partial \rho}, \quad D_{\theta}=\frac{\partial}{\partial \theta},
$$


for polar coordinates $\rho, \theta$; if $\phi=\left\{\phi_{\alpha}^{j}\right.$ and $\psi=\left\{\psi_{\alpha}^{j}\right\}$, for $j=1,2, \cdots n$, then $\phi \cdot \psi=\sum_{\alpha} \sum_{j=1}^{N} C_{\alpha} \phi_{\alpha}^{j} \psi_{\alpha}^{j}$, where $C_{\alpha}=|\alpha| ! / \alpha_{1} !, \cdots, \alpha_{v} ! . \quad|\phi|^{2}=\phi \cdot \phi . \quad u, j$ $=\partial u / \partial x^{j} \quad u_{, \alpha}=D^{\alpha} u \quad \alpha=\left(\alpha_{1}, \cdots, \alpha_{v}\right) ; \quad \nabla^{m} u=\left\{u_{\alpha_{\alpha}}^{i}\right\}, \quad|\alpha|=m, \quad i=1,2$, $\cdots, N$, if $u=\left(u^{1} \cdots u^{N}\right) \cdot \int_{G} f(x) d x=$ Lebesgue integral with respect to $\nu$-dimensional Euclidean measure $d x . H_{2}^{m}(G)$ equals the "closure of $C^{\infty}(G)$ functions with respect to the norm $(0.1)$ " (more precisely, equivalence classes of such functions).

$$
\begin{aligned}
\|u\|_{2, G}^{m} & =\left[(u, u)_{2, G}^{m}\right]^{1 / 2}, \\
(u, v)_{2, G}^{m} & =\int_{G}\left(\sum_{j=0}^{m} \nabla^{j} u \cdot \nabla^{j} v\right) d x .
\end{aligned}
$$

$H_{2}^{m}(G)$ is known to be a Hilbert Space (see, e.g., [4]). $H_{20}^{m}(G)=$ "closure of $C_{\mathrm{c}}^{\infty}(G)$ " in the norm $(0.1)$, where $C_{\mathrm{c}}^{\infty}(G)=$ functions of $G$ which vanish outside some compact set $\Lambda \subset G$. We will say that an element $u$ of $H_{2}^{m}(G)$ has a certain point property if some representative $\bar{u}$ of $u$ has it. Summation convention is employed without mention. $D^{m}(u, G)=\int_{G}\left|\nabla^{m} u\right|^{2} d x$; $\left|\nabla^{m} a\right|^{2}=$ weighted sum of squares of components of $u$.

Theorem 0.1. Let $G \subset E$, be bounded and suppose

$$
f(x, z, p) \geqq s|p|^{2}+t
$$

(for notation see paragraph below Lemma 2.3) and is convex in $p$ for each $(x, z)$. Let $F$ be a family of functions $u$ of class $H_{2}^{m}(G)$ which is (i) closed with respect to weak convergence in $H_{2}^{m}(G)$, (ii) contains a function $u_{1}$ for which $I\left(u_{1}, G\right)<\infty$, (iii) is such that for each $u \in F$ we may find a $u^{*}$ in a family $F^{*}$ with $\left\|u^{*}\right\|_{2, G}^{m} \leqq B$ on $F^{*}$, so that $u^{*}-u \in H_{20}^{m}(G)$. Then $I(u, G)$ takes on its minimum in $F$.

Proof. For $m=1$ see [1]. The proof for any $m$ is a simple reworking of the case $m=1$ see, e.g., [5].

The following are standard results. Complete proofs may be found in [4] or [5].

TheOREM 0.2. $C^{\infty}\left(\overline{B_{r}}\right)$ is dense in $H_{2}^{m}\left(B_{r}\right)$.

Lemma 0.1. Suppose $u(x) \in H_{2}^{1}(G), v(x) \in C^{0}(G)$ and $\nabla u(x)=v(x)$ a.e. Then there is a function $\bar{u}(x)=u(x)$ a.e. such that $\bar{u}(x) \in C^{1}(G)$ and $\nabla \bar{u}(x)$ $=v(x)$.

We use the following form of Sobolev's lemma.

Lemma 0.2. If $u \in \bigcap_{m=0}^{\infty} H_{2}^{m}\left(B_{r}\right)$ then $u \in C^{\infty}\left(B_{r}\right)$.

Lemma 0.3. Suppose $\phi \in H_{2}^{m}(G), e_{j}$ is the $x^{j}$ unit vector, and $D \subset G$ is such that

$$
\phi_{h}=h^{-1}\left[\phi\left(x+h e_{j}\right)-\phi(x)\right]
$$


is drfinec for $0<|h|<h_{0}$. Then $\phi_{h} \rightarrow \phi{ }_{j}$ in $H_{2}^{m-1}(D)$.

LEMMA 0.4. Bounded sets in $H_{2}^{m}(G)$ are relatively compact with respect to weak convergence.

LemMa 0.5. If $\left\{u_{n}\right\}$ converges weakly to $u$ in $H_{2}^{m}(G)$ then $\left\{u_{n}\right\}$ converges strongly to $u$ in $H_{2}^{m-1}(G)$.

LEMmA 0.6. If $u \in H_{2}^{m}\left(B_{R}\right)$, then there exists a constant $\eta(m, v)$ and $a$ function $U \in H_{20}^{m}\left(B_{2 R}\right)$ such that $U(x)=u(x)$ for $x \in B_{R}$ and

$$
D^{m}\left(U, B_{2 R}\right) \leqq \eta(m, \nu) \sum_{j=0}^{m} R^{2(j-m)} \int_{B_{R}}\left|\nabla^{j} u\right|^{2} d x .
$$

1. An estimate for polyharmonic functions.

Lemma 1.1. Suppose $u$ and $v \in C^{\infty}\left(B_{r}-\left\{x_{0}\right\}\right)$. Then, if $0<\rho<r, 0$ $\leqq|\alpha| \leqq m, 0 \leqq|\beta| \leqq m$, and $m=0,1, \cdots$, it follows that

$$
D_{\rho \theta}^{\alpha}(u, 1) D_{\rho \theta}^{\beta}(v, 1)+D_{\rho \theta}^{\alpha}(u, 2) D_{\rho \theta}^{\beta}\left(v,,_{2}\right)=b_{\gamma \delta} D_{\rho \theta}^{\gamma} u D_{\rho \theta}^{\delta} v,
$$

where $b_{\gamma \delta}=c_{\gamma \delta} \rho^{-p}, p=p(\gamma, \delta)$ is a non-negative integer, and the sum in (1.1) is taken for $0 \leqq|\gamma| \leqq m+1,0 \leqq|\delta| \leqq m+1$, and $c_{\gamma \delta}$ are constants.

Proof. Express $u_{, 1}$ and $u_{, 2}$ in polar coordinates, then use Leibniz's rule for differentiating products. Now let ()$_{j}=d^{j}() / d \theta^{j}$. The result is

$$
\begin{aligned}
& D_{\rho \theta}^{\alpha}(u, 1)=A_{\alpha}^{j}(u)(\cos \theta)_{j}-B_{\alpha}^{j}(u)(\sin \theta)_{j}, \\
& D_{\rho \theta}^{\alpha}\left(u,,_{2}\right)=B_{\alpha}^{j}(u)(\cos \theta)_{j}+A_{\alpha}^{j}(u)(\sin \theta)_{j},
\end{aligned}
$$

where $j$ is summed from 1 to $\alpha_{2}$ and

$$
\begin{aligned}
& A_{\alpha}^{j}(u)=C_{\alpha}^{j} D_{\rho \theta}^{\gamma} u, \quad \gamma=\gamma(\alpha, j), \quad 0 \leqq|\gamma| \leqq m+1, \\
& B_{\alpha}^{j}(u)=K_{\alpha \delta}^{j} \rho^{-q(\delta)} D_{\rho \theta}^{\delta} u, \quad 0 \leqq|\delta| \leqq m+1 .
\end{aligned}
$$

Hence, recalling that $(\cos \theta)_{j}=\cos (\theta+j \pi / 2)$ and $(\sin \theta)_{j}=\sin (\theta+j \pi / 2)$ we find

$$
\begin{aligned}
& D_{\rho \theta}^{\alpha}\left(u,,_{1}\right) D_{\rho \theta}^{j}(v, 1)+D_{\rho \theta}^{\alpha}\left(u,,_{2}\right) D_{\rho \theta}^{\beta}\left(v,{ }_{2}\right) \\
& \quad=\lambda_{j k}\left[A_{\alpha}^{j}(u) A_{\beta}^{k}(v)+B_{\alpha}^{j}(u) B_{\beta}^{k}(v)\right]+\mu_{j k}\left[A_{\alpha}^{j}(u) B_{\beta}^{k}(v)-B_{\alpha}^{j}(u) A_{\beta}^{k}(v)\right],
\end{aligned}
$$

where

$$
\begin{aligned}
& \lambda_{j k}=(\cos \theta)_{j}(\cos \theta)_{k}+(\sin \theta)_{j}(\sin \theta)_{k}=\cos \left[(j-k) \frac{\pi}{2}\right], \\
& \mu_{j k}=(\sin \theta)_{j}(\cos \theta)_{k}-(\cos \theta)_{j}(\sin \theta)_{k}=\sin \left[(j-k) \frac{\pi}{2}\right] .
\end{aligned}
$$

Thus $\lambda_{j k}$ and $\mu_{j k}$ are constants. The proof is completed by substituting (1.3) in (1.4).

LEMMA 1.2. Under the hypotheses of Lemma 1.1, we may find $a_{\alpha \beta}=c_{\alpha \beta}(m) \rho^{-p}$ such that 


$$
\nabla^{m} u \cdot \nabla^{m} v=a_{\alpha \beta} D_{\rho \theta}^{\alpha} u D_{\rho \theta}^{\beta} v, \quad|\alpha| \leqq m, \quad|\beta| \leqq m,
$$

where $p=p(\alpha, \beta)$ is a non-negative integer, and $c_{\alpha \beta}$ are constants.

Proof. Since

$$
\nabla^{m+1} u \cdot \nabla^{m+1} v=\nabla^{m}(u, 1) \cdot \nabla^{m}(v, 1)+\nabla^{m}(u, 2) \cdot \nabla^{m}(v, 2)
$$

we may use induction on $m$ and Lemma 1.1.

Definition 1.1. For $u \in C\left(B_{r}\right)$ and $\rho>0$ we define

$$
s_{N}(u, \rho, \theta)=a_{0} / 2+\sum_{n=1}^{N}\left[a_{n} \cos n \theta+b_{n} \sin n \theta\right],
$$

where

$$
\begin{aligned}
& a_{n}=a_{n}(u, \rho, \theta)=\frac{1}{\pi} \int_{0}^{2 \pi} u(\rho, \theta) \cos n \theta d \theta, \\
& b_{n}=b_{n}(u, \rho, \theta)=\frac{1}{\pi} \int_{0}^{2 \pi} u(\rho, \theta) \sin n \theta d \theta .
\end{aligned}
$$

Lemma 1.3. Suppose $u \in C^{\infty}\left(\overline{B_{r}}\right)$ and $0<\rho \leqq r$. Then for each $\rho$ and $m=0,1, \ldots$ we find

$$
\int_{0}^{2 \pi}\left|\nabla^{m}\left(u-s_{N}\right)\right|^{2} d \theta=\int_{0}^{2 \pi}\left|\nabla^{m} u\right|^{2} d \theta-\int_{0}^{2 \pi}\left|\nabla^{m} s_{N}\right|^{2} d \theta
$$

and

$$
\lim _{N \rightarrow \infty} \int_{0}^{2 \pi}\left|\nabla^{m}\left(u-s_{N}\right)\right|^{2} d \theta=0 .
$$

Proof. Evidently, $\partial s_{N} / \partial \theta=s_{N}\left(u_{\theta}, \rho, \theta\right)$ and $\partial s_{N} / \partial \rho=s_{N}\left(u_{\rho}, \rho, \theta\right)$ hence, by induction, we infer that

$$
D_{\rho \theta}^{\alpha} s_{N}(u, \rho, \theta)=s_{N}\left(D_{\rho \theta}^{\alpha}, \rho, \theta\right) .
$$

From Lemma 1.2 and (1.8) we conclude that

$$
\int_{0}^{2 \pi}\left|\nabla^{m}\left(u-s_{N}\right)\right|^{2} d \theta=a_{\alpha \beta} \int_{0}^{2 \pi}\left[D_{\rho \theta}^{\alpha} u-s_{N}\left(D_{\rho \theta}^{\alpha} u\right)\right]\left[D_{\rho \theta}^{\beta} u-s_{N}\left(D_{\rho \theta}^{\beta} u\right)\right] d \theta,
$$

where $\alpha$ and $\beta$ are summed over $|\alpha| \leqq m$ and $|\beta| \leqq m$. (1.7) follows by first applying the Schwarz inequality to the right side of (1.9) followed by the Riesz-Fischer theorem in $L_{2}[(0,2 \pi)]$ applied to the functions $D_{\rho \theta}^{\alpha} u$, and $D_{\rho \theta}^{\beta} u$. From a Fourier expansion for $f$ and $g$ in $L_{2}$ we see that

$$
\int_{0}^{2 \pi}\left(f-s_{N}(f)\right)\left(g-s_{N}(g)\right) d \theta=\int_{0}^{2 \pi} f g d \theta-\int_{0}^{2 \pi} s_{N}(f) s_{N}(g) d \theta .
$$

Apply this result to the right side of (1.9), taking $f=D_{\rho \theta}^{\alpha} u$ and $g=D_{\rho \theta}^{\beta} u$. 
Then, using (1.8) to commute operations, a backwards application of Lemma 1.2 proves (1.6).

TheOREM 1.1. If $u \in C^{\infty}\left(\overline{B_{r}}\right)$ then

$$
\int_{B_{r}}\left|\nabla^{m}\left(u-s_{N}\right)\right|^{2} d x<\infty \text {. }
$$

Moreover,

$$
\lim _{N \rightarrow \infty} \int_{B_{r}}\left|\nabla^{m}\left(u-s_{N}\right)\right|^{2} d x=0
$$

and

$$
\int_{B_{r}}\left|\nabla^{m}\left(u-s_{N}\right)\right|^{2} d x=\int_{B_{r}}\left|\nabla^{m} u\right|^{2} d x-\int_{B_{r}}\left|\nabla^{m} s_{N}\right|^{2} d x .
$$

Proof. First use (1.6) to establish dominated convergence of the integrals in question in polar coordinates, then integrate (1.6) and (1.7).

Definition 1.2. $u \in T\left(B_{r}\right)$ if and only if for some $a_{n}$ and $b_{n}$ in $C^{\infty}[(0, r)]$

$$
u=a_{0}(\rho) / 2+\sum_{n=1}^{N}\left[a_{n}(\rho) \cos n \theta+b_{n}(\rho) \sin n \theta\right], \quad 0<\rho \leqq r .
$$

LEMma 1.4. $T\left(B_{r}\right) \cap H_{2}^{m}\left(B_{r}\right)$ is a dense linear subset of $H_{2}^{m}\left(B_{r}\right)$.

Proof. Theorem 1.1 shows that $T\left(B_{r}\right) \cap H_{2}^{m}\left(B_{r}\right)$ is dense in $C^{\infty}\left(\bar{B}_{r}\right)$ with respect to the $H_{2}^{m}\left(B_{r}\right)$ norm. Theorem 0.2 asserts that $C^{\infty}\left(\overline{B_{r}}\right)$ is dense in $H_{2}^{m}\left(B_{r}\right)$.

Definition 1.3. If $u \in T\left(B_{r}\right)$ we define $J_{T}: T\left(B_{r}\right) \rightarrow T\left(B_{r}\right)$ by the formula

$$
J_{T} u=a_{0}(\rho) / 2+\sum_{n=1}^{N}\left[b_{n}(\rho) \cos n \theta-a_{n}(\rho) \sin n \theta\right] .
$$

Lemma 1.5. If $u \in T\left(B_{r}\right)$ then for $0<\rho<r$

$$
\int_{0}^{2 \pi}\left(J_{T} u\right)\left(J_{T} v\right) d \theta=\int_{0}^{2 \pi} u \cdot v d \theta .
$$

Proof. By direct computation.

Lemma 1.6. If $u \in T\left(B_{r}\right)$ then so is $D_{\rho \theta}^{\alpha} u$, and $J_{T}\left(D_{\rho \theta}^{\alpha} u\right)=D_{\rho \theta}^{\alpha}\left(J_{T} u\right)$.

Proof. If $D_{\rho \theta}^{\alpha}=\partial / \partial \theta$ or $\partial / \partial \rho$ we may compute directly. The general case follows by induction.

LEMma 1.7. If $u \in T\left(B_{r}\right) \cap H_{2}^{m}\left(B_{r}\right)$ then

$$
\int_{B_{r}}\left|\nabla^{j}\left(J_{T} u\right)\right|^{2} d x=\int_{B_{r}}\left|\nabla^{j} u\right|^{2} d x, \quad j=0,1, \cdots, m .
$$

Proof. Use Lemma 1.2 to evaluate $\left|\nabla^{j}\left(J_{T} u\right)\right|^{2}$. In the resulting sum 
commute $D_{\rho \theta}^{\alpha}$ and $J_{T}$ using Lemma 1.6. Integrate from 0 to $2 \pi$, then apply Lemma 1.5. To complete the proof, reapply Lemma 1.2 , multiply by $\rho$ and integrate from 0 to $r$.

THEOREM 1.2. There exists a unique bounded linear transformation

$$
J: L_{2}\left(B_{r}\right) \rightarrow L_{2}\left(B_{r}\right)
$$

whose restriction to $T\left(B_{r}\right) \cap L_{2}\left(B_{r}\right)$ is equal to $J_{T}$. For every integer $m \geqq 0$, the restriction of $J$ to $H_{2}^{m}\left(B_{r}\right)$ is, in the norm of that space, a bounded linear transformation. In addition, $J$ has the properties enumerated below.

$$
J^{2} u=\frac{1}{\pi} \int_{0}^{2 \pi} u d \theta-u \quad(\text { a.e. in } \rho) .
$$

(1.13) As an operator on $H_{2}^{m}\left(B_{r}\right), m \geqq 2, J$ commutes with $\Delta$.

(1.14) $J$ is an isometric transformation on each space $H_{2}^{m}\left(B_{r}\right), m=0,1,2, \cdots$.

(1.15) If $u \in H_{2}^{m}\left(B_{r}\right)$ and $j=0,1, \cdots, m$ then

$$
\int_{0}^{2 \pi}\left|\nabla^{j}(J u)\right|^{2} d \theta=\int_{0}^{2 \pi}\left|\nabla^{j} u\right|^{2} d \theta,
$$

for almost every $\rho: 0<\rho<r$.

(1.16) If $u \in L_{2}\left(B_{r}\right)$ then for almost every $\rho, u$ is given by its Fourier series in $\theta$. Hence, $J u$ is given by the conjugate series for almost all $\rho$. That is, with respect to convergence in $L_{2}[(0,2 \pi)]$, we have

$$
J u=a_{0}(\rho) / 2+\sum_{n=1}^{\infty}\left[b_{n}(\rho) \cos n \theta-a_{n}(\rho) \sin n \theta\right] .
$$

Proof. From Lemma $1.4, J_{T}$ is densely defined. From Lemma $1.7, J_{T}$ is isometric on $T\left(B_{r}\right) \cap H_{2}^{m}\left(B_{r}\right) \quad(m=0,1, \ldots)$. Hence, a unique isometric extension exists for each $H_{2}^{m}\left(B_{r}\right)$. The extensions all agree since $H_{2}^{m}$ convergence implies $H_{2}^{l}$ convergence for $l \leqq m$. (1.12) and (1.13) are easily checked on $T\left(B_{r}\right)$. They persist because of the continuity of $J$ for all the spaces $H_{2}^{m}$ and that of $\Delta$ on $H_{2}^{m}(m \geqq 2)$. (1.14) implies that

$$
\int_{B_{r}}\left|\nabla^{j}(J u)\right|^{2} d x=\int_{B_{r}}\left|\nabla^{j} u\right|^{2} d x
$$

for $u \in H_{2}^{m}\left(B_{r}\right)$ and $j=0,1, \cdots, m$. (1.15) then follows by differentiating with respect to $\rho$. (1.16) follows from (1.15) for $j=0$, an application of Fubini's theorem in polar coordinates, and $L_{2}[(0,2 \pi)]$ convergence of a Fourier series.

REMARK. If $J_{\rho}$ and $J_{r}(\rho<r)$ are the maps of the preceding theorem defined for $B_{\rho}$ and $B_{r}$, respectively, then $J_{\rho}$ is a restriction of $J_{r}$. This is easily established by approximating on $B_{r}$ by functions of $T\left(B_{r}\right)$, then cutting them down to $T\left(B_{\rho}\right)$. We often denote $J u$ by $\bar{u}$. 
Theonem 1.3. $C^{\infty}\left(B_{r}\right), C_{c}^{\infty}\left(B_{r}\right)$, and $H_{20}^{m}\left(B_{r}\right)$ are invariant under $J$.

Proof. If $u \in C^{\infty}\left(B_{r}\right)$ then $u \in \bigcap_{m=0}^{\infty} H_{2}^{m}\left(B_{\rho}\right)$ for $0<\rho<r$. Thus, $J u$ $\in \bigcap_{m=0}^{\infty} H_{2}^{m}\left(B_{\rho}\right)$. It follows from Sobolev's lemma that $J u \in C^{\infty}\left(B_{\rho}\right)$, $0<\rho<r$, and hence $J u \in C^{\infty}\left(B_{r}\right)$. Now if $u \in C_{c}^{\infty}\left(B_{r}\right)$ it follows from (1.14) for $m=0$ that $J u$ vanishes outside the support of $u$. The remaining assertion follows by approximation.

Theorem 1.4. If $u \in H_{2}^{2}\left(B_{r}\right)$ is harmonic in $B_{r}$ then so is $\bar{u}$. Moreover, $u$ and $\bar{u}$ satisfy the Cauchy-Riemann equations $u,_{1}-\bar{u}_{,_{2}}=0, u,_{2}+\bar{u}_{,_{1}}=0$.

Proof. $\Delta \bar{u}=\overline{\Delta u}=\overline{0}=0$ hence $\bar{u}$ is harmonic. It is well known that

$$
u=a_{0} / 2+\sum_{n=1}^{\infty}(\rho / r)^{n}\left(a_{n} \cos n \theta+b_{n} \sin n \theta\right),
$$

hence

$$
\bar{u}=a_{0} / 2+\sum_{n=1}^{\infty}(\rho / r)^{n}\left(b_{n} \cos n \theta-a_{n} \sin n \theta\right) .
$$

Evidently $u_{\rho}=\rho^{-1} \bar{u}_{\theta}$ and $\bar{u}_{\rho}=-\rho^{-1} u_{\theta}$. But these are the polar CauchyRiemann equations.

THEOREM 1.5. If $u^{*} \in H_{2}^{m}(G)$ and $G$ is bounded, then there is a unique function $u \in H_{2}^{m}(G) \cap C^{\infty}(G)$ such that $u-u^{*} \in H_{20}^{m}(G)$ and $\Delta^{m} u=0$ in $G$.

Proof. See [4].

Lemma 1.8. If $v$ and $w \in C^{\infty}([0,2 \pi])$ and $v(0)=v(2 \pi), w(0)=w(2 \pi)$, then

$$
\int_{0}^{2 \pi} v w_{\theta} d \theta \leqq \frac{1}{2} \int_{0}^{2 \pi}\left(v_{\theta}^{2}+w_{\theta}^{2}\right) d \theta
$$

Proof. Expand in a Fourier series and use the inequality $a b \leqq \frac{1}{2}\left(a^{2}+b^{2}\right)$.

Lemma 1.9. Suppose $v, w \in C^{\infty}\left(\bar{B}_{r}\right)$. Then

$$
\begin{aligned}
\int_{B_{r}}\left\{|\nabla v|^{2}+|\nabla w|^{2}\right\} d x & \\
& \leqq \int_{B_{r}}\left[\left(v,,_{1}-w, 2\right)^{2}+\left(v,,_{2}+w,_{1}\right)^{2}\right] d x+r^{2} \int_{\partial B_{r}}\left(|\nabla v|^{2}+|\nabla w|^{2}\right) d \theta .
\end{aligned}
$$

Proof. From Green's theorem and a change to polar coordinates in the resulting boundary integral we find

$$
\begin{aligned}
\int_{B_{r}}\left(|\nabla v|^{2}\right. & \left.+|\nabla w|^{2}\right) d x \\
& =\int_{B_{r}}\left[(v, 1-w, 2)^{2}+\left(v, 2+w, s_{1}\right)^{2}\right] d x+2 \int_{\partial B_{r}} v w_{\theta} d \theta .
\end{aligned}
$$

An application of Lemma 1.8 yields 


$$
2 \int_{\partial B_{r}} v w_{\theta} d \theta \leqq \int_{\partial B_{r}}\left(v_{\theta}{ }^{2}+w_{\theta}{ }^{2}\right) d \theta \leqq r^{2} \int_{\partial B_{r}}\left(|\nabla v|^{2}+|\nabla w|^{2}\right) d \theta
$$

from which the desired result follows.

Lemma 1.10. Suppose $v, w \in H_{2}^{m}\left(B_{R}\right)$. For each $r: 0<r \leqq R$ let $V_{r}$ and $W_{r}$ be functions in $H_{2}^{m}\left(B_{r}\right)$, such that $\left(V_{r}-v\right)$ and $\left(W_{r}-w\right) \in H_{20}^{m}\left(B_{r}\right)$. Then, for almost all $r: 0<r \leqq R$, we have $\nabla^{m} v$ and $\nabla^{m} w \in L_{2}[(0,2 \pi)]$, and for such $r$

$$
\begin{aligned}
& \int_{\dot{B}_{r}}\left(\left|\nabla^{m} V_{r}\right|^{2}+\left|\nabla^{m} W_{r}\right|^{2}\right) d x \\
& \leqq \int_{B_{r}}\left(\left|\nabla^{m-1} F_{r}\right|^{2}+\left|\nabla^{m-1} G_{r}\right|^{2}\right) d x+r^{2} \int_{\partial B_{r}}\left(\left|\nabla^{m} v\right|^{2}+\left|\nabla^{m} w\right|^{2}\right) d \theta,
\end{aligned}
$$

where $F_{r}=V_{r, 1}-W_{r, 2}$ and $G_{r}=V_{r, 2}+W_{r, 1}$.

Proof. Let $v_{n}, w_{n} \in C^{\infty}\left(\bar{B}_{R}\right)$ be such that $v_{n} \rightarrow v$ and $w_{n} \rightarrow w$ in $H_{2}^{m}\left(B_{R}\right)$. By passing to a subsequence if necessary we conclude from the theorems of Fubini and Fatou that (a.e. in $r$ )

$$
\lim _{n \rightarrow \infty} \int_{\partial B_{r}}\left|\nabla^{m}\left(v-v_{n}\right)\right|^{2} d \theta=\lim _{n \rightarrow \infty} \int_{\partial B_{r}}\left|\nabla^{m}\left(w-w_{n}\right)\right|^{2} d \theta=0 .
$$

Since $\left(V_{r}-v\right)$ and $\left(W_{r}-w\right)$ are in $H_{20}^{m}\left(B_{r}\right)$ we may find $C_{c}^{\infty}\left(B_{r}\right)$ functions $\phi_{n r} \rightarrow V_{r}-v, \psi_{n r} \rightarrow W_{r}-w$ in $H_{2}^{m}\left(B_{r}\right)$. Let $V_{n r}=v_{n}+\phi_{n r}, W_{n r}=w_{n}$ $+\psi_{n r}$. Evidently, $V_{n r} \rightarrow V_{r}, W_{n r} \rightarrow W_{r}$ in $H_{2}^{m}\left(B_{r}\right)$. We apply Lemma 1.9 to find that

$$
\begin{aligned}
\int_{B_{r}}\left(\left|\nabla^{m} V_{n r}\right|^{2}\right. & +\left|\nabla^{m} W_{n r}\right|^{2} d x \\
= & \int_{B_{r}} \sum C_{\alpha}\left(\left|\nabla V_{n r, \alpha}\right|^{2}+\left|\nabla W_{n r, \alpha}\right|^{2}\right) d x \\
\leqq & \int_{B_{r}} \sum C_{\alpha}\left\{\left[\left(V_{n r, 1}-W_{n r, 2}\right)_{\alpha}\right]^{2}+\left[\left(V_{n r, 2}+W_{n r, 1}\right)_{\alpha}\right]^{2}\right\} d x \\
& +r^{2} \int_{\partial B_{r}} \sum C_{\alpha}\left(\left|\nabla v_{n, \alpha}\right|^{2}+\left|\nabla w_{n, \alpha}\right|^{2}\right) d \theta,
\end{aligned}
$$

where the sums are taken for $|\alpha|=m-1$. From (1.18) and the $H_{2}^{m}$ convergence of $V_{n r}$ and $W_{n r}$ to $V_{r}$ and $W_{r}$ the lemma follows.

THEOREM 1.6. Suppose $u \in H_{2}^{m}\left(B_{R}\right)$ and $U_{r}$ is the function of Theorem 1.5 for $G=B_{r}$ (i.e., $\left.\Delta^{m} U_{r}=0, \quad U_{r}-u \in H_{20}^{m}\left(B_{r}\right)\right)$. Then for $l(m)=2^{m}-1$ and almost all $r: 0<r \leqq R$,

$$
\int_{B_{r}}\left|\nabla^{m} U_{r}\right|^{2} d x \leqq l(m) r \int_{\partial B_{r}}\left|\nabla^{m} u\right|^{2} d s .
$$

Proof. Let $\bar{u}$ be the conjugate of $u$ in $B_{R}$ and let $\bar{U}_{r}$ be the conjugate of $U_{r}$ in $B_{r}$. From Theorems 1.2 and $1.3 \bar{u} \in H_{2}^{m}\left(B_{R}\right), \bar{U}_{r}-\bar{u} \in H_{20}^{m}\left(B_{r}\right)$, and $\Delta^{m} \bar{U}_{r}=0$. For $m=1$ take $V_{r}=U_{r}, W_{r}=\bar{U}_{r}, v=u$, and $w=\bar{u}$ in (1.17). 
From Thıoren 1.4 $F_{r}=G_{r}=0$ and thus (a.e. in $r, 0<r \leqq R$ )

$$
\int_{B_{r}}\left(\left|\nabla U_{r}\right|^{2}+\left|\nabla \bar{U}_{r}\right|^{2}\right) d x \leqq r^{2} \int_{\partial B_{r}}\left(|\nabla u|^{2}+|\nabla \bar{u}|^{2}\right) d \theta .
$$

Applying (1.14) and (1.15) to the left and right sides of (1.20) respectively we find $(1.19)_{1}$. Assuming $(1.19)_{m-1}$, we again take $V_{r}=U_{r}, W_{r}=\bar{U}_{r}$, $v=u, w=\bar{u}$ in (1.17) to find after an application of (1.14) and (1.15) to the left- and right-hand sides as before, that for almost all $r: 0<r \leqq R$

$$
\begin{aligned}
\int_{B_{r}}\left|\nabla^{m} U_{r}\right|^{2} d x & \\
& \leqq \frac{1}{2} \int_{B_{r}}\left(\left|\nabla^{m-1} F_{r}\right|^{2}+\left|\nabla^{m-1} G_{r}\right|^{2}\right) d x+r^{2} \int_{\partial B_{r}}\left|\nabla^{m} u\right|^{2} d \theta .
\end{aligned}
$$

However, since $\Delta^{m-1} U_{r}$ and $\Delta^{m-1} \bar{U}_{r}=\overline{\Delta^{m-1} U_{r}}$ are conjugate harmonic we observe that

$$
\begin{aligned}
& \Delta^{m-1} F_{r}=\left(\Delta^{m-1} U_{r}\right)_{1}-\left(\Delta^{m-1} \bar{U}_{r}\right)_{2}=0 \\
& \Delta^{m-1} G_{r}=\left(\Delta^{m-1} U_{r}\right)_{2}+\left(\Delta^{m-1} \bar{U}_{r}\right)_{1}=0 .
\end{aligned}
$$

If we let $f=u,,_{1}-\bar{u}_{, 2}$ and $g=u,,_{2}+\bar{u}_{1}$ it is easy to check that $f, g \in H_{2}^{m-1}\left(B_{R}\right)$ and $\left(F_{r}-f\right),\left(G_{r}-g\right) \in H_{20}^{m-i}\left(B_{r}\right)$. Thus we may apply $(1.19)_{m-1}$ to the pairs $F_{r}, f$ and $G_{r}, g$ which, combined with (1.21), yields (a.e. in $r$ )

$$
\begin{aligned}
\int_{B_{r}}\left|\nabla^{m} U_{r}\right|^{2} d x & \\
& \leqq \frac{1}{2} l(m-1) r \int_{\partial B_{r}}\left(\left|\nabla^{m-1} f\right|^{2}+\left|\nabla^{m-1} g\right|^{2}\right) d s+r \int_{\partial B_{r}}\left|\nabla^{m} u\right|^{2} d s .
\end{aligned}
$$

If we substitute for $f$ and $g$ their values in terms of $u$ and $\bar{u}$ and use elementary estimates, this becomes (a.e. in $r$ )

$$
\begin{aligned}
\int_{B_{r}}\left|\nabla^{m} U_{r}\right|^{2} d x \\
\quad \leqq \frac{1}{2} l(m-1) r \int_{\partial B_{r}} 2\left(\left|\nabla^{m} u\right|^{2}+\left|\nabla^{m} \bar{u}\right|^{2}\right) d s+r \int_{\partial B_{r}}\left|\nabla^{m} u\right|^{2} d s .
\end{aligned}
$$

Finally, another application of (1.15) to the right side of (1.22) yields (a.e. in $r$ )

$$
\int_{B_{r}}\left|\nabla^{m} U_{r}\right|^{2} d x \leqq[2 l(m-1)+1] r \int_{\partial B_{r}}\left|\nabla^{m} u\right|^{2} d s
$$

which is just $(1.19)_{m}$.

\section{Dirichlet growth and regularity.}

Lemma 2.1. Suppose a tensor $v(x) \in H_{2}^{1}(G)$ and, for some $L$ independent of $r$. 


$$
\int_{B_{r}}|\nabla v|^{2} d x \leqq L^{2}\left(\frac{r}{R}\right)^{2 \lambda}, \quad 0 \leqq r \leqq R,
$$

whenever $B_{R} \subset G$. Then

$$
\left|v\left(x_{2}\right)-v\left(x_{1}\right)\right| \leqq C_{1}(\lambda) L R^{-\lambda}\left|x_{1}-x_{2}\right|^{\lambda}, \quad 0 \leqq\left|x_{2}-x_{1}\right| \leqq R,
$$

for every pair of points $\left(x_{1}, x_{2}\right)$ whose joining segment is at distance $>R$ from $\partial G ;\left(C_{1}(\lambda)=2^{2-\lambda} \pi^{-1 / 2} \lambda^{-1}\right)$.

Proof. See [3].

Lemma 2.2. Suppose a tensor $u(x) \in H_{2}^{m}(G)$ and

$$
\int_{B_{r}}\left|\nabla^{m} u\right|^{2} d x \leqq L^{2}\left(\frac{r}{R}\right)^{2 \lambda}, \quad 0 \leqq r \leqq R,
$$

whenever $B_{R} \subset G$. Then, $u \in C^{m-1}(G)$ and

$$
\left|\nabla^{m-1} u\left(x_{2}\right)-\nabla^{m-1} u\left(x_{1}\right)\right| \leqq C_{1}(\lambda) L R^{-\lambda}\left|x_{2}-x_{1}\right|^{\lambda}
$$

whenever $0 \leqq\left|x_{1}-x_{2}\right| \leqq R$, for every pair of points $\left(x_{1}, x_{2}\right)$ in $G$ whose joining segment is at distance $>R$ from $\partial G$.

Proof. Apply Lemma 2.1 to $v=\nabla^{m-1} u$. The proof is completed by applying Lemma $0.1 m-1$ times.

Lemma 2.3. Suppose $v \in H_{2}^{m}\left(B_{R}\right), \int_{0}^{R} s^{-1} \psi(s) d s<\infty$ and

$$
D^{m}\left(v, B_{r}\right) \leqq K D^{m}\left(v_{r}, B_{r}\right)+\psi(r), \quad 0<r \leqq R,
$$

for every $v_{r}: v-v_{r} \in H_{20}^{m}\left(B_{r}\right)$. Then for $0<r \leqq R$

$$
D^{m}\left(v, B_{r}\right) \leqq D^{m}\left(v, B_{R}\right)\left(\frac{r}{R}\right)^{\mu}+\mu r^{\mu} \int_{r}^{R} \rho^{-\mu-1} \psi(\rho) d \rho,
$$

where $\mu=[K l(m)]^{-1}$ (see Theorem 1.6). vand $v_{r}$ may be tensors.

Proof. Let $\phi(r)=D^{m}\left(v, B_{r}\right)$, and for each $v^{i}$ take $u=v^{i}$ and define $v_{r}^{i}$ $=U_{r}$ as in Theorem 1.6. Letting $v_{r}=\left\{v_{r}^{i}\right\}$ and recalling (1.19) $m$ we find (a.e. in $r$ )

$$
D^{m}\left(v_{r}, B_{r}\right) \leqq l(m) r \phi^{\prime}(r)
$$

and hence from (2.5) we find a.e. for $0<r \leqq R$

$$
\phi(r) \leqq \mu^{-1} r \phi^{\prime}(r)+\psi(r) .
$$

The desired result follows after multiplication of $(2.7)_{\rho}$ by the integrating factor $\rho^{-\mu-1}$ and integrating from $r$ to $R$.

In what follows, we consider functions $f(x, z, p)$ where $x=\left(x^{1}, x^{2}\right)$, $\alpha=\left(\alpha_{1}, \alpha_{2}\right),|\alpha|=\alpha_{1}+\alpha_{2} ; z=\left\{z_{\alpha}^{i}\right\} i=1,2, \cdots N, 0 \leqq|\alpha| \leqq m-1 ; \quad p=\left\{p_{\alpha}^{i}\right\}$ $i=1,2, \cdots N,|\alpha|=m$. If $u \in H_{2}^{m}(G)$ we define $z(x)=\left\{u_{, \alpha}^{i}(x)\right\} i=1,2$, $\cdots N, \quad 0 \leqq|\alpha| \leqq m-1$ and $p(x)=\left\{u_{\gamma_{\alpha}}^{i}(x)\right\} \quad i=1,2, \cdots N,|\alpha|=m$. In 
order to insure the existence of a minimum for $I(u, G)=\int_{G} f(x, z(x), p(x)) d x$ $\mathrm{w}$ : mpose (see Theorem 0.1)

$$
f(x, z, p) \text { is continuous in }(x, z, p)
$$

and convex in $p$ for all $(x, z)$. Moreover, there exist constants $s, S, k$ such that $0<s \leqq S$ and

$$
s|p|^{2}-k \leqq f(x, z, p) \leqq S|p|^{2}+k .
$$

LeмmA 2.4. Suppose $f$ satisfies (2.8), and $u_{0}$ minimizes $I(u, G)$ among all $u: u-u_{0} \in H_{20}^{m}(G)$. Then, if $B_{r} \subset G$ it follows that

$$
D^{m}\left(u_{0}, B_{r}\right) \leqq \frac{S}{s} D^{m}\left(u_{r}, B_{r}\right)+\frac{2 \pi k}{s} r^{2}
$$

for all $u_{r}: u_{r}-u_{0} \in H_{20}^{m}\left(B_{r}\right)$.

Proof. Since $H_{20}^{m}\left(B_{r}\right) \subset H_{20}^{m}(G)$ we conclude that $u_{0}$ is minimal for $I\left(u, B_{r}\right)$ among all $u_{r}: u_{r}-u_{0} \in H_{20}^{m}\left(B_{r}\right)$. Thus $I\left(u_{0}, B_{r}\right) \leqq I\left(u_{r}, B_{r}\right)$, and if we apply $(2.8)_{2}$ to this inequality, (2.9) follows.

TheOREм 2.1. Under the hypotheses of Lemma $2.4 u_{0} \in C_{\mu}^{m-1}(G)$. $\left(u_{0}\right.$ $\in C^{m-1}(G), \nabla^{m-1} u_{0}$ Hölder continuous for every $\left.D: \bar{D} \subset G\right)$.

Proof. The "Dirichlet growth" necessary to apply Lemma 2.2 follows by successively applying (2.9) and Lemma 2.3 with $v=u_{0}$. Thus we easily obtain a local Hölder estimate. The global condition follows from some simple metric space lemmas.

Definition 2.1. All integrals in this definition are over $G$.

$A(u, v ; G)=\int v_{, \alpha}^{j} a_{j k}^{\alpha \beta}(x) u^{b}{ }_{\beta} d x ;|\alpha|=|\beta|=m, a_{j k}^{\alpha \beta}=a_{k j}^{\beta \alpha} . \quad B(u, v ; G)=\int v,_{\alpha}^{j}$ $b_{j k}^{\alpha \beta}(x) u^{k}{ }_{, \beta} d x$, where $0 \leqq \min (|\alpha|,|\beta|)<\max (|\alpha|,|\beta|)=m, b_{j k}^{\alpha \beta}=b_{j k}^{\beta \alpha} . C(u, v ; G)$ $=\int v_{\alpha}^{j} c_{j k}^{\alpha \beta}(x) u,_{\beta}^{k} d x ; 0 \leqq \max (|\alpha|,|\beta|)<m, c_{j k}^{\alpha \beta}=c_{j k}^{\beta \alpha} . \quad E(v ; G)=\int e_{j}^{\alpha} v_{, \alpha}^{j} d x ;$ $|\alpha|=m . F(v ; G)=\int f_{j}^{\alpha} v_{\alpha}^{j} d x ; 0 \leqq|\alpha|<m$. $a, b, c, e$, and $f$ are measurable, $e \in L_{2}(G)$ and

$$
\begin{gathered}
s_{1}|\pi|^{2} \leqq a_{j k}^{\alpha \beta} \pi_{\alpha}^{j} \pi_{\beta}^{k} \leqq S_{1}|\pi|^{2}, \quad 0<s_{1} \leqq S_{1} . \\
\int \chi_{r}(x)\left(|b|^{2}+|c|+|f|\right) d x \leqq S_{2}^{2} r^{2 \lambda}
\end{gathered}
$$

for all circles $B_{r .} \chi_{r}$ is the characteristic function of $B_{r .} I(u, v ; G)=A(u, v ; G)$ $+B(u, v ; G)+C(u, v ; G) . \quad L(v ; G)=E(v ; G)+F(v ; G)$. Finally, we consider solutions of the equation

$$
I(u, v ; G)=L(v),
$$

that is, functions $u \in H_{2}^{m}(G)$ such that (2.12) holds for every $v \in H_{20}^{m}(G)$.

LEMмA 2.5. If $u \in H_{20}^{m}(G), G_{r}=G \cap B_{r}$,

$$
\int_{G_{r}}|f| d x \leqq L r^{2 \lambda}
$$


for every circle $B_{r}$, and $G \subset B\left(x_{1}, R\right)$, then $f \cdot z(x)$ and $f|z(x)|^{2}$ are in $L_{1}(G)$ and

$$
\begin{array}{cc}
\int_{G_{r}}|f \cdot z| d x \leqq C_{2} g^{\mu}\left[D^{m}(u, G)\right]^{1 / 2} r^{2 \lambda-\mu}, & 0<\mu<\lambda, \\
\int_{G_{r}}|f||z|^{2} d x \leqq C_{3} g^{\mu} D^{m}(u, G) r^{2 \lambda-\mu}, & 0<\mu<\lambda,
\end{array}
$$

where $\pi g^{2}=|G|, z(x)=\left\{u_{, \alpha}^{j}\right\}, j=1,2, \cdots, N, 0 \leqq|\alpha|<m . C_{i}=L K_{i}(\lambda, \mu, m, R)$, $i=2,3$, remains bounded for $R \rightarrow 0$. ( $u$ and $f$ may be tensors.)

Proof. $(2.14)_{1}$ and $(2.15)_{1}$ are Lemmas 4.4 and 4.5 of [3]. Since $z(x)$ $\in H_{20}^{1}$ we apply these results to $f \cdot z$ and $f \cdot z^{2}$, respectively. The lower order terms which then appear may be handled by Poincarés inequality [3, Theorem 1.11].

LeMma 2.5a. If $u \in H_{2}^{m}\left(B_{r}\right)$ and $f \in L_{1}\left(B_{R}\right)$ satisfies (2.13) for $G=B_{R}$, then $f \cdot z(x)$ and $f z(x)^{2}$ are in $L_{1}\left(B_{R}\right)$ and

$$
\begin{array}{cc}
\int_{G_{r}}|f \cdot z| d x \leqq C_{4}\|u\|_{2, R}^{m} r^{2 \lambda-\mu}, & 0<\mu<\lambda, \\
\int_{G_{r}}|f||z|^{2} d x \leqq C_{5}\left(\|u\|_{2, R}^{m}\right)^{2} r^{2 \lambda-\mu}, & 0<\mu<\lambda,
\end{array}
$$

$C_{i}=L K_{i}(\lambda, \mu, m, R), i=4,5$.

Proof. Let $U \in H_{20}^{m}\left(B_{2 R}\right)$ be the function of Lemma 0.6. Extend $f$ to be zero outside of $B_{R}$, then apply (2.14) and (2.15) to $f \cdot Z$ and $f Z^{2}$. An application of Lemma 0.6 completes the proof.

Lemma 2.6. There exists an $R_{0}>0$ depending only on $m, s_{1}, S_{2}$, and $\lambda$, for which $0<R \leqq R_{0}$ and $B_{R} \subset G$ together imply that

$$
\left(\frac{s_{1}}{2}\right) D^{m}\left(u, B_{R}\right) \leqq I\left(u, u ; B_{R}\right) \leqq\left(S_{1}+\frac{s_{1}}{2}\right) D^{m}\left(u, B_{R}\right),
$$

for all $u \in H_{20}^{m}\left(B_{R}\right)$.

Proof. From the Schwarz inequality, Lemma 2.5 and (2.11)

$$
\begin{gathered}
\left|B\left(u, u ; B_{R}\right)\right| \leqq 2 \overline{C_{3}}\left[D^{m}\left(u, B_{R}\right)\right] R^{2 \lambda}, \\
\left|C\left(u, u ; B_{R}\right)\right| \leqq \overline{C_{3}}\left[D^{m}\left(u, B_{R}\right)\right] R^{2 \lambda},
\end{gathered}
$$

where $\overline{C_{3}}=\inf C_{3}$ for $\mu: 0<\mu<\lambda$. Since $\overline{C_{3}}$ remains bounded as $R \rightarrow 0$ we may find $R_{0}$ so small that $|B|+|C| \leqq\left(s_{1} / 2\right) D^{m}\left(u, B_{R}\right)$. The lemma follows from $(2.10)$.

Lemma 2.7. If $u, v \in C^{m}(G) G \subset E_{v}$ then

$$
D^{\alpha}(u \cdot v)=\sum_{\alpha=\beta+\gamma}\left(\begin{array}{c}
\alpha \\
\beta
\end{array}\right) D^{\beta} u D^{\gamma} v ;\left(\begin{array}{c}
\alpha \\
\beta
\end{array}\right)=\left(\begin{array}{c}
\alpha_{1} \\
\beta_{1}
\end{array}\right) \ldots\left(\begin{array}{c}
\alpha_{\nu} \\
\beta_{\nu}
\end{array}\right) .
$$


$u$ and $v$ may be tensors.

Proof. By induction on $\nu$.

Lemma 2.8. $\left|\nabla^{m+1}\left(\left|x-x_{0}\right|\right)\right| \leqq F(\nu, m)\left|x-x_{0}\right|^{-m}, x \neq x_{0}$ and $m=0,1 \cdots$.

Proof. $\phi(y)=\left|\nabla^{m+1}(|y|)\right|$ is positively homogeneous of degree $-m$, hence let $F(\nu, m)=\sup _{|y|=1} \phi(y)$.

LEMMA 2.9. If $h(t) \in C_{c}^{\infty}\left(E_{1}\right), \quad \xi(x)=h\left[(R-r)^{-1}\left(\left|x-x_{0}\right|-r\right)\right]$ and $r \geqq R / 2>0$ then for constants $h_{j}=h_{j}(\nu)$

$$
\left|\nabla^{j} \xi(x)\right| \leqq h_{j}(R-r)^{-j}, \quad j=0,1, \cdots,
$$

whenever $\left|x-x_{0}\right| \geqq r$.

Proof. By induction on $j$, with the aid of Lemmas 2.7 and 2.8. The induction hypothesis is to be applied to $h^{\prime}\left[(R-r)^{-1}\left(\left|x-x_{0}\right|-r\right)\right]$.

LeMmA 2.10. If $h$ and $\xi$ are as in Lemma 2.9 and in addition $h(t)=1$ for $t \leqq 1 / 4, h(t)=0$ for $t \geqq 3 / 4$, and $h(t)$ is monotone then $\xi \in C_{c}^{\infty}\left(B_{R}\right)$ and (2.19) holds for all $x$ if $r \geqq R / 2>0$.

Lemma 2.11. If $\xi$ is the function of Lemma 2.10 then $D^{\alpha}\left(\xi^{m}\right)=\xi^{m-|\alpha|} \phi_{\alpha}(\xi)$ and

$$
\left|\phi_{\alpha}(\xi)\right| \leqq K(m, \nu)(R-r)^{-|\alpha|}
$$

for $0 \leqq|\alpha| \leqq m$ and $r \geqq R / 2>0$.

Proof. By iteration of Lemma 2.7 and application of (2.19).

Lemma 2.12. Suppose that $\phi \in C_{c}^{\infty}(G), G \subset E_{v}$ and $u \in H_{2}^{m}(G)$ then $\phi u$ $\in H_{20}^{m}(G)$ and has compact support in G. Moreover, the formula of Lemma 2.7 continues to hold.

Proof. By approximation to $u$.

Theorem 2.2. Suppose $B_{R} \subset G, 0<R \leqq R_{0}, u \in H_{2}^{m}\left(B_{R}\right)$ and satisfies (2.12) for each $v \in H_{20}^{m}\left(B_{R}\right)$. If $a, b, c, e, f$ are as in Definition 2.1 then for $0<r<R$

$$
D^{m}\left(u, B_{r}\right) \leqq C_{6}\left[\left(\|u\|_{2, R}^{m-1}\right)^{2}+\left(\|e\|_{2, R}^{0}\right)^{2}+1\right],
$$

$C_{6}=C_{6}\left(r, R, m, \lambda, s_{1}, S_{1}, S_{2}\right)$.

Proof. For $\xi$ as in Lemma 2.10, $U(x)=\xi^{m} u(x)$ and $v(x)=\xi^{m} U(x)$ are in $H_{20}^{m}\left(B_{R}\right)$. Since $D^{m}\left(u, B_{r}\right) \leqq D^{m}\left(u, B_{R / 2}\right)$ for $r<R / 2$ we assume $r \geqq R / 2$. From Lemmas 2.7 and 2.11 we find for $|\alpha| \leqq m$, and $J=\{(\beta, \gamma, \delta)$ : $\beta+\gamma+\delta=\alpha,|\beta|>0\}$ that $v_{, \alpha}^{j}=\xi^{m}\left[U_{, \alpha}^{j}+\sum_{J} \xi^{m-|\beta+\gamma|} \phi_{\beta}(\xi) \phi_{\gamma}(\xi) u_{,}^{j}\right]$. Collecting terms and applying the estimate of Lemma 2.11.

$$
v_{, \alpha}^{j}=\xi^{m}\left[U_{, \alpha}^{j}+p_{\alpha}^{\gamma}(\xi) u_{\alpha}^{j}\right], \quad 0 \leqq|\gamma|<|\alpha| \leqq m,|p| \leqq P(m, r, R) .
$$

Analogously, 
(2.23) $\xi^{m} u_{, \alpha}^{j}=U_{, \alpha}^{j}+q_{\alpha}^{\gamma}(\xi) u_{\gamma}^{j}, \quad 0 \leqq|\gamma|<|\alpha| \leqq m, \quad|q| \leqq Q(m, r, R)$.

Hence, $I\left(u, v ; B_{R}\right)=I\left(U, U ; B_{R}\right)+I_{1}$, as follows from (2.12), (2.22) and (2.23) where

$$
\begin{aligned}
& I_{1}=\int_{B_{R}}\left[a_{j k}^{\alpha \beta} \cdot(*)+b_{j k}^{\alpha \beta} \cdot(*)+c_{j k}^{\alpha \beta}(*)\right] d x-L\left(v ; B_{R}\right), \\
& *=p_{\alpha}^{\gamma} u_{\gamma}^{j} U_{, \beta}^{k}+q_{\beta}^{\gamma} u_{\gamma}^{k} U_{, \alpha}^{j}+p_{\alpha}^{\gamma} q_{\beta}^{\delta} u_{\gamma}^{j} u_{, \delta}^{k} .
\end{aligned}
$$

$I_{1}$ may be estimated using $A(u, v) \leqq[A(u, u)]^{1 / 2}[A(v, v)]^{1 / 2}$, and the ordinary Schwarz inequality, followed by the estimates of Lemmas 2.5 and 2.5a. The inequality $2(a b) \leqq \epsilon a^{2}+\epsilon^{-1} b^{2}$ is used to handle terms involving $D^{m}\left(U, B_{R}\right)$. We find

$$
\left|I_{1}\right| \leqq \epsilon C_{6}^{\prime} D^{m}\left(U, B_{R}\right)+C_{6}^{\prime \prime}\left[\left(\|u\|_{2, R}^{m-1}\right)^{2}+\left(\|e\|_{2, R}^{0}\right)^{2}+1\right],
$$

$C_{6}^{\prime}=C_{6}^{\prime}\left(r, R, m, \lambda, S_{1}, S_{2}\right), C_{6}^{\prime \prime}=C_{6}^{\prime \prime}\left(r, R, m, \lambda, S_{1}, S_{2}, \epsilon\right)$. Choosing, e.g., $\in C_{6}^{\prime}$ $\leqq s_{1} / 4$ we see that

$$
0=I\left(u, v ; B_{R}\right)-L\left(v, B_{R}\right) \geqq I\left(U, U ; B_{R}\right)-\left|I_{1}\right| \geqq\left(s_{1} / 2\right) D^{m}\left(U, B_{R}\right)-\left|I_{1}\right| .
$$

The theorem follows from (2.24) since $D^{m}\left(u, B_{r}\right) \leqq D^{m}\left(U, B_{R}\right)$.

LEMma 2.13. Suppose $0<R \leqq R_{0}, B_{R} \subset G, u \in H_{20}^{m}\left(B_{R}\right)$ and (2.12) holds for all $v \in H_{20}^{m}\left(B_{R}\right)$. Then if

$$
\int_{B_{r}}|e|^{2} d x \leqq L^{2}\left(\frac{r}{\delta}\right)^{2 r}, \quad 0 \leqq r \leqq \delta ; \quad \delta=R-\left|x_{1}-x_{0}\right| ; \quad 0<\tau<\frac{\lambda}{2} ;
$$

for every circle $B_{r}=B\left(x_{1}, r\right)$ contained in $B_{R}=B\left(x_{0}, R\right)$ it follows that

$$
D^{m}\left(u, B_{r}\right) \leqq\left(\frac{S_{1}}{s_{1}}\right) D^{m}\left(u_{r}, B_{r}\right)+C_{7}\left(\frac{r}{\delta}\right)^{2 r}, \quad 0 \leqq r \leqq \delta,
$$

whenever $u-u_{r} \in H_{20}^{m}\left(B_{r}\right) . \quad C_{7}=C_{7}\left(m, s_{1}, S_{1}, S_{2}, \lambda, \tau, L, R,\|u\|_{2, R}^{m}\right)$ is a quadratic function of its last variable.

Proof. Define

$$
\begin{aligned}
& E_{j}^{\alpha}=-\sum_{|\beta|<m} b_{j k}^{\alpha \beta} u_{, \beta}^{k}+e_{j}^{\alpha}, \\
& F_{j}^{\alpha}=-\sum_{|\beta|=m} b_{j k}^{\alpha \beta} u_{\beta}^{k}-\sum_{|\beta|<m} c_{j k}^{\alpha \beta} u_{, \beta}^{k}+f_{j}^{\alpha} .
\end{aligned}
$$

From the Schwarz inequality and Lemma 2.5a, we see that

$$
\begin{gathered}
\|E\|_{2, r}^{0} \leqq C_{5}^{1 / 2}\|u\|_{2, R}^{m} r^{\lambda-\mu / 2}+L\left(\frac{r}{\delta}\right)^{r}, \\
\int_{B_{r}}|F| d x \leqq S_{2}^{2} r^{2 \lambda}+\|u\|_{2, R}^{m} S_{2} r^{\lambda}+C_{4}\|u\|_{2, R}^{m} r^{2 \lambda-\mu} .
\end{gathered}
$$

Choosing, e.g, $\mu=\lambda / 2$ it follows that

$$
\|E\|_{2, r}^{0} \leqq\left(C_{7}^{1}\left(\lambda, \mu, R, m, S_{2}\right)\|u\|_{2, R}^{m}+L\right)\left(\frac{r}{\delta}\right)^{r}
$$




$$
\int_{i_{r}}|F| d x \leqq\left[C_{7}^{2}\left(S_{2}, R, \lambda\right)+\|u\|_{2, R}^{m} C_{7}^{3}\left(S_{2}, R, \lambda, m\right)\right] r^{\lambda} .
$$

If we define

$$
L^{*}{ }_{u}\left(v ; B_{r}\right)=-\int_{B_{r}}\left[\sum_{|\alpha|=m} E_{j}^{\alpha} v_{\alpha}^{j}+\sum_{|\alpha|<m} F_{j}^{\alpha} v_{,_{\alpha}}^{j}\right] d x .
$$

Then

$$
A\left(u, v ; B_{r}\right)=L^{*}{ }_{u}\left(v, B_{r}\right) \quad \text { for all } v \in H_{20}^{m}\left(B_{r}\right) .
$$

Since $A\left(u, v ; B_{r}\right)$ is a scalar product, equivalent to the usual one on $H_{20}^{m}\left(B_{r}\right)$, the Riesz representation theorem asserts the existence of $U_{r} \in H_{20}^{m}\left(B_{r}\right)$ which satisfies (2.29). Then, estimating the right side of (2.29) and employing (2.10), (2.27) and (2.28), we find

$$
D^{m}\left(U_{r}, B_{r}\right) \leqq s_{1}^{-2}\left[\|E\|_{2, r}^{0}+C_{4} r^{\lambda}\right] \text {. }
$$

Now, being careful to check the definition of $C_{4}$ (we have taken $\mu=\lambda / 2$ ) it follows that

$$
D^{m}\left(U_{r}, B_{r}\right) \leqq s_{1}^{-2}\left[C_{7}^{4}+C_{7}^{5}\|u\|_{2, R}^{m}\right]\left(\frac{r}{\delta}\right)^{2 r},
$$

$C_{7}^{i}=C_{7}^{i}\left(m, s_{1}, S_{2}, \lambda, \tau, L, R\right), \quad i=4,5$.

Let $V_{r}=u-U_{r}$. From the Pythagorean property of $A, V_{r}$ minimizes $A$ among all $u_{r}: u-u_{r} \in H_{20}^{m}\left(B_{r}\right)$ and

$$
A\left(u, u ; B_{r}\right)=A\left(U_{r}, U_{r} ; B_{r}\right)+A\left(V_{r}, V_{r} ; B_{r}\right)
$$

so that

$$
D^{m}\left(u, B_{r}\right) \leqq s_{1}^{-1} A(u, u) \leqq \frac{S_{1}}{s_{1}} D^{m}\left(U_{r}, B_{r}\right)+\frac{S_{1}}{s_{1}} D^{m}\left(u_{r}, B_{r}\right) .
$$

The proof of the lemma is completed by applying (2.30) to the right side.

3. Final regularity results for the variational problem. In addition to the previous notation and restrictions on $f$ (see \$2) we now require

$$
f \in C^{\prime \prime} \text { for all }(x, z, p) \text {. }
$$

(3.2) There are functions $s_{1}(R), S_{1}(R)$ defined for all $R \geqq 0$ such that $0<s_{1}(R) \leqq S_{1}(R)$ and

for all $(x, z, p):|x|^{2}+|z|^{2} \leqq R^{2}$.

$$
s_{1}(R)|\pi|^{2} \leqq f_{p_{\alpha}^{j} p_{\beta}^{k}} \pi_{\alpha}^{j} \pi_{\beta}^{k} \leqq S_{1}(R)|\pi|^{2}
$$

(3.3) There is a function $S_{2}(R)$ defined for all $R \geqq 0$ such that $\sum_{i=1}^{5} \sigma_{i}$ $\leqq S_{2}(R)|p|^{2}$ for all $(x, z, p):|x|^{2}+|z|^{2} \leqq R^{2}$, where

$$
\begin{gathered}
\sigma_{1}=\sum f_{p_{\alpha} z_{\beta}}^{2}, \sigma_{2}=\sum f_{p_{\alpha} x}^{j}, \sigma_{3}^{j}=\sum\left|f_{z_{\alpha} z_{\beta}}^{j}\right|, \\
\sigma_{4}=\sum\left|f_{z_{\alpha} x}^{i l}\right|, \sigma_{5}=\sum f_{p_{\alpha}}^{2} .
\end{gathered}
$$


All indices except $l$ are summed, and over their full range.

Theorem 3.1. Suppose $u \in H_{2}^{m}(G) \cap C^{m-1}(G)$ and $\zeta \in C_{c}^{m}(G)$. If $\psi(\lambda)$ $=I(u+\lambda \zeta, G)$ then

$$
\psi^{\prime}(0)=\int_{G} \zeta_{,_{\alpha}}^{j} f_{z_{\alpha}}^{i}[x, z(x), p(x)] d x+\int_{G} \pi_{\alpha}^{i} f_{p_{\alpha}}^{i}[x, z(x), p(x)] d x .
$$

Proof. Expand $f[x, z(x)+\lambda \zeta(x), p(x)+\lambda \pi(x)]$ about $(x, z(x), p(x))$ using Taylor's formula with integral remainder. Then integrate over $G$ and calculate the difference quotient $[\psi(\lambda)-\psi(0)] / \lambda$. Because of the continuity of $\dot{u}$, smoothness of $\zeta$, and conditions (3.1), (3.2), (3.3) we obtain dominated convergence for the sequence of integrands in question for $|\lambda| \leqq 1$. Allowing $\lambda \rightarrow 0$ completes the proof.

THEOREM 3.2. Suppose $u \in H_{2}^{m}(G) \cap C^{m-1}(G), \psi^{\prime}(0)=0$ and $u$ has the growth properties of Lemma 2.4. Then $u \in C_{\mu}^{m}(G) \cap H_{2}^{m+1}(D)$. for each $D$ : $\bar{D} \subset G$.

Proof. We show that the conclusions of the theorem hold in some neighborhood of each point in $G$. It is then easy to prove the global result.

First suppose $B\left(x_{0}, R_{1}\right) \subset G, 0<R_{3}<R_{2}<R_{1}$, and $R_{2}-R_{3}=R_{1}-R_{2}$ $=h_{0} \leqq d\left(B_{R_{1}}, \partial G\right)$. If $v \in C_{c}^{\infty}\left(B_{R_{3}}\right)$, we let $\zeta=v\left(x-h e_{\gamma}\right)-v(x)$, where $e_{\gamma}$ is the unit vector in the $x_{\gamma}$ direction. Then

$$
0=\int_{B_{R_{2}}}\left\{f_{z_{\alpha}}^{j}\left[v_{\gamma_{\alpha}}^{j}\left(x-h e_{\gamma}\right)-v_{\alpha_{\alpha}}^{j}(x)\right]+f_{p_{\alpha}}^{j}\left[v_{, \alpha}^{j}\left(x-h e_{\gamma}\right)-v_{\gamma_{\alpha}}^{j}(x)\right]\right\} d x .
$$

Now, break up (3.4) into four integrals; translate variables in those two which involve $x-h e_{\gamma}$; recombine and factor $v_{\alpha_{\alpha}}^{j}$. Using the device $g(1)$ $-g(0)=\int_{0}^{1} g^{\prime}(t) d t$ we find that $u_{h}=h^{-1}\left[u\left(x+h e_{\gamma}\right)-u(x)\right]$ satisfies (2.12) with $G=B\left(x_{0}, R_{3}\right)$ for all $v \in C_{c}^{\infty}\left(B_{R_{3}}\right)$ and

$$
\begin{aligned}
a_{h j k}^{\alpha \beta} & =\int_{0}^{1} f_{z_{\alpha} p_{\beta}^{k}}^{j k}\left[P_{t}\right] d t, \quad b_{h j k}^{\alpha \beta}=-\int_{0}^{1} f_{z_{\alpha} p_{\beta}^{k}}^{j}\left[P_{t}\right] d t, \\
c_{h j k}^{\alpha \beta} & =\int_{0}^{1} f_{z_{\alpha} z_{\alpha}^{k}}^{j}\left[P_{t}\right] d t, \quad e_{h_{j}}^{\alpha}=-\int_{0}^{1} f_{p_{\alpha}^{j} x^{\gamma}}\left[P_{t}\right] d t \\
f_{h j}^{\alpha} & =-\int_{0}^{1} f_{z_{\alpha}^{j}{ }^{\gamma}}{ }^{\gamma}\left[P_{t}\right] d t, \\
P_{t} & =\left[x+t h e_{\gamma},(1-t) z(x)+t z\left(x+h e_{\gamma}\right),(1-t) p(x)+t p\left(x+h e_{\gamma}\right)\right] .
\end{aligned}
$$

Using conditions (3.1), (3.2), (3.3) and the continuity of $|z(x)|$ we may verify

$$
s_{1}(Q)|\pi|^{2} \leqq a_{h j k}^{\alpha \beta}(x) \pi_{\alpha}^{j} \pi_{\beta}^{k} \leqq S_{1}(Q)|\pi|^{2}
$$

and for $|h| \leqq h_{0}, G_{r}=B\left(x_{1}, r\right) \cap B\left(x_{0}, R_{3}\right)$

$$
\int_{G_{r}}\left(\left|b_{h}\right|^{2}+\left|c_{h}\right|+\left|f_{h}\right|+\left|e_{h}\right|^{2}\right) d x \leqq \bar{S}_{2}^{2} r^{2 \lambda}
$$


The bounds are independent of $h$ but depend on $u$. In making the estimate (3.6) we must use the fact that the integral growth condition does not depend on the center of the circle. If we let $R_{4}=\min \left(R_{3}, R_{0}\right)$, where $R_{0}$ is the radius of Lemma 2.6, we find that $u_{h}$ satisfies the requirements of Theorem 2.2 on $B_{R_{4}}$. From Lemma 0.3 we find that $u_{h} \rightarrow u_{x \gamma}$ in $H_{2}^{m-1}\left(B_{R_{4}}\right)$. All the more, the $H_{2}^{m-1}\left(B_{R_{4}}\right)$ norms are bounded for some $h_{1}: 0<|h| \leqq h_{1}$ $\leqq h_{0}$. From Theorem 2.2 we infer that the $H_{2}^{m}\left[B\left(x_{0}, R_{5}\right)\right]$ norms of $u_{h}$ are uniformly bounded for any $R_{5}<R_{4}$. Hence for a subsequence of $h$ tending to zero, the weak relative compactness implies that $u_{h} \neg u^{*}$, $u^{*} \in H_{2}^{m}\left(B_{R_{5}}\right)$ (Lemma 0.4). Since weak $H_{2}^{m}$ convergence implies strong $H_{2}^{m-1}$ convergence (Lemma 0.5) we conclude that $u_{x \gamma}=u^{*} \in H_{2}^{m}\left(B_{R_{5}}\right)$, i.e., $u \in H_{2}^{m+1}\left(B_{R_{5}}\right)$.

Finally, the uniform boundedness of the $H_{2}^{m}$ norms on $B_{R_{5}}$ together with Lemma 2.13 yields a uniform Hölder condition for $\nabla^{m-1} u_{h_{n}}$ for $|h| \leqq h_{1}$ on $B_{R_{6}}, R_{6}<R_{5}$. From equicontinuity, a subsequence converges uniformly, hence, $\nabla^{m-1} u_{x \gamma}$ satisfies the same Hölder condition on $B\left(x_{0}, R_{6}\right)$.

\section{BIBLIOGRAPHY}

1. C. B. Morrey, Jr., Multiple integral problems in the calculus of variations and related topics, Univ. Calif. Publ. Math. (N. S.) 1 (1943), 1-130.

2. $ـ$ Second order elliptic systems of differential equations, Annals of Mathematics Studies No. 33, Princeton Univ. Press, Princeton, N. J., 1954, 101-159.

3. _ Multiple integral problems in the calculus of variations and related topics. Ann. Scuola Norm. Sup. Pisa (3) 14 (1960), 1-61.

4. Lecture notes on the theory of elliptic partial differential equations, mimeographed notes, Univ. of Chicago, Chicago, Ill., 1960.

5. Julius Smith, Variational problems of higher order for double integrals, Dissertation, Univ. ff California, Berkeley, Calif., 1962.

UNIVERSITY OF ILLINOIS,

URBANA, ILLINOIS 